\title{
Extended supersymmetry of semichiral sigma
} models in $4 D$

\section{UIf Lindström}

Department of Physics and Astronomy, Division of Theoretical Physics, Uppsala University, Box 516, SE-751 20 Uppsala, Sweden

E-mail: ulf.lindstrom@physics.uu.se

ABSTRACT: Briefly: using a novel $(1,1)$ superspace formulation of semichiral sigma models with $4 D$ target space, we investigate if an extended supersymmetry in terms of semichirals is compatible with having a $4 D$ target space with torsion.

In more detail: semichiral sigma models have $(2,2)$ supersymmetry and Generalized Kähler target space geometry by construction. They can also support $(4,4)$ supersymmetry and Generalized Hyperkähler geometry, but when the target space is four dimensional indications are that the geometry is restricted to Hyperkähler. To investigate this further, we reduce the model to $(1,1)$ superspace and construct the extra (on-shell) supersymmetries there. We then find the conditions for a lift to $(2,2)$ super space and semichiral fields to exist. Those conditions are shown to hold for Hyperkähler geometries. The $\mathrm{SU}(2) \otimes$ U(1) WZW model, which has $(4,4)$ supersymmetry and a semichiral description, is also investigated. The additional supersymmetries are found in $(1,1)$ superspace but shown not to be liftable to a $(2,2)$ semichiral formulation.

Keywords: Extended Supersymmetry, Differential and Algebraic Geometry, Sigma Models

ARXIV EPRINT: 1411.3906 


\section{Contents}

1 Introduction 1

2 Background 2

2.1 Semichiral sigma models 2

2.2 Extra SUSY 3

$3 N=(4,4)$ in $N=(1,1)$ superspace $\quad 4$

$3.1 N=(2,2) \quad 5$

$3.2 N=(4,4) \quad 6$

4 The $S^{3} \times S^{1}$ model $\quad 7$

4.1 Duality 7

4.2 The geometry 8

$\begin{array}{lll}4.3 & \text { Additional SUSY in }(1,1) & 10\end{array}$

4.3.1 Deriving the transformations on the $(1,1)$ coordinate fields $\quad 10$

$\begin{array}{lll}\text { 4.3.2 } & \left(X^{L}, X^{R}\right) \text { coordinates } & 12\end{array}$

5 Examples $\quad \mathbf{1 3}$

$\begin{array}{llr}5.1 \text { Hyperkähler } & 13\end{array}$

$\begin{array}{lll}5.2 & \mathrm{SU}(2) \otimes \mathrm{U}(1) & 13\end{array}$

6 Discussion 14

$\begin{array}{ll}\text { A Duality in }(1,1) & 15\end{array}$

$\begin{array}{lll}\text { B An alternative dual form } & 17\end{array}$

\section{Introduction}

Generalized Kähler geometry is efficiently probed by $(2,2)$ supersymmetric sigma models in $D=2$, [1]. Of particular interest for the present investigation is the symplectic case, i.e., sigma models that depend on semichiral superfields only. Additional supersymmetries for these models were discussed in [2], and in [3]. In the latter article focus is on four-dimensional target spaces and it is shown that a very general ansatz for additional supersymmetries leads to an on-shell extended supersymmetry and restricts the target space geometry to be hyperkähler.

In [3] this is seen as a shortcoming of the ansatz, since it is argued that the $\mathrm{SU}(2) \otimes \mathrm{U}(1)$ WZW model of [4] constitutes a counterexample. It has nonzero torsion and when coordinatized by chiral and twisted chiral superfields it has "manifest" $(4,4)$ supersymmetry. 
It further has a dual semichiral description [5] which is then expected to also display the $(4,4)$ supersymmetry. ${ }^{1}$

In this paper we investigate the possibility that the $(2,2)$ semichiral conditions are incompatible with "manifest" $(4,4)$ transformations. $^{2}$ To study this problem, we descend to $(1,1)$ superspace and develop an on-shell formalism for the extra super symmetries, a formulation which retains the relation to $(2,2)$ semichirals. We test this $(1,1)$ formalism on the second supersymmetry (which is non-manifest in $(1,1)$ ) and then apply it to a hyperkähler geometry which is shown to satisfy the conditions for having a $(2,2)$ semichiral realisation, as expected from [3].

We also derive the extra supersymmetries for the WZW model [4] in $(1,1)$ superspace in the relevant coordinates. When subjected to the same test they fail to satisfy some of the conditions. This leads to the surprising conclusion that $(4,4)$ supersymmetry in a $(1,1)$ formulation of a $(2,2)$ sigma model with on-shell supersymmetry is incompatible with the introduction of the $(2,2)$ auxiliary fields.

\section{Background}

\subsection{Semichiral sigma models}

Consider a generalized Kähler potential [1] with one left- and one right semichiral field and their complex conjugates, $K\left(\mathbb{X}^{L}, \mathbb{X}^{R}\right)$, where $L=(\ell, \bar{\ell})$ and $R=(r, \bar{r})$. The action,

$$
S=\int d^{2} x d^{2} \theta d^{2} \bar{\theta} K\left(\mathbb{X}^{L}, \mathbb{X}^{R}\right)
$$

has manifest $N=(2,2)$ supersymmetry. The supersymmetry algebra is defined in terms of the anti-commutator of the covariant supersymmetry derivatives as

$$
\left\{\mathbb{D}_{ \pm}, \overline{\mathbb{D}}_{ \pm}\right\}=i \partial_{\underline{\underline{\#}}}
$$

and the semichiral fields are defined by their chirality constraints as [7]

$$
\overline{\mathbb{D}}_{+} \mathbb{X}^{\ell}=0, \quad \overline{\mathbb{D}}_{-} \mathbb{X}^{r}=0 .
$$

The geometry of the model is bi-hermitean $[7,8]$, governed by two complex structures $J^{(+)}$and $J^{(-)}$that both preserve the metric $\mathbb{G}$

$$
J^{( \pm) t} \mathbb{G} J^{( \pm)}=\mathbb{G}
$$

as well as by an anti-symmetric $B$-field whose field strength $H$ enters in the form of torsion in the covariant constancy conditions

$$
0=\nabla^{( \pm)} J^{( \pm)}=\left(\partial+\Gamma^{(0)} \pm \frac{1}{2} H \mathbb{G}^{-1}\right) J^{( \pm)}
$$

\footnotetext{
${ }^{1}$ By "manifest" we shall mean "as realised by transformations of $(2,2)$ superfields".

${ }^{2}$ Another case of supersymmetries being obstructed occurs when dualisation is along isometries that do not commute with the extra super symmetries. This leads to nonlocal realisations of the extra susys in the dual model [6]. Here, however, the extra susys commute with the isometry used in dualising.
} 
where $\Gamma^{(0)}$ is the Levi-Civita connection. These conditions identify the geometry as bihermitean [8], or generalized Kähler geometry (GKG) [9].

The fact that our superfields are semichiral specifies the GKG as being of symplectic type where the metric $\mathbb{G}$ and the $\mathbb{B}$-field take the form ${ }^{3}$

$$
\begin{aligned}
& \mathbb{G}=\Omega\left[J^{(+)}, J^{(-)}\right] \\
& \mathbb{B}=\Omega\left\{J^{(+)}, J^{(-)}\right\} .
\end{aligned}
$$

The matrix $\Omega$ is defined as

$$
\Omega=\frac{1}{2}\left(\begin{array}{cc}
0 & K_{L R} \\
-K_{R L} & 0
\end{array}\right)
$$

and the submatrix $K_{L R}$ is the Hessian

$$
K_{L R}=\left(\begin{array}{cc}
K_{\ell r} & K_{\ell \bar{r}} \\
K_{\bar{\ell} r} & K_{\bar{\ell} \bar{r}}
\end{array}\right) .
$$

An additional condition results from the target space being four-dimensional and reads $[7]$

$$
\left\{J^{(+)}, J^{(-)}\right\}=2 c, \quad \Rightarrow \mathbb{B}=2 c \Omega,
$$

where in general $c$ is a function of the coordinates.

\subsection{Extra SUSY}

In [3] it is shown that a general ansatz for $(4,4)$ susy in a semichiral sigma model

$$
\begin{aligned}
& \delta \mathbb{X}^{\ell}=\bar{\epsilon}^{+} \overline{\mathbb{D}}_{+} f\left(\mathbb{X}^{L}, \mathbb{X}^{R}\right)+g\left(\mathbb{X}^{\ell}\right) \bar{\epsilon}^{-} \overline{\mathbb{D}}_{-} \mathbb{X}^{\ell}+h\left(\mathbb{X}^{\ell}\right) \epsilon^{-} \mathbb{D}_{-} \mathbb{X}^{\ell} \\
& \delta \mathbb{X}^{\bar{\ell}}=\epsilon^{+} \mathbb{D}_{+} \bar{f}\left(\mathbb{X}^{L}, \mathbb{X}^{R}\right)+\bar{g}\left(\mathbb{X}^{\bar{\ell}}\right) \epsilon^{-} \mathbb{D}_{-} \mathbb{X}^{\bar{\ell}}+\bar{h}\left(\mathbb{X}^{\bar{\ell}}\right) \bar{\epsilon}^{-} \overline{\mathbb{D}}_{-} \mathbb{X}^{\bar{\ell}} \\
& \delta \mathbb{X}^{r}=\bar{\epsilon}-\overline{\mathbb{D}} \bar{f}_{-}\left(\mathbb{X}^{L}, \mathbb{X}^{R}\right)+\tilde{g}\left(\mathbb{X}^{r}\right) \bar{\epsilon}^{+} \overline{\mathbb{D}}_{+} \mathbb{X}^{r}+\tilde{h}\left(\mathbb{X}^{r}\right) \epsilon^{+} \mathbb{D}_{+} \mathbb{X}^{r} \\
& \delta \mathbb{X}^{\bar{r}}=\epsilon^{-} \mathbb{D}_{-} \overline{\tilde{f}}\left(\mathbb{X}^{L}, \mathbb{X}^{R}\right)+\overline{\tilde{g}}\left(\mathbb{X}^{\bar{r}}\right) \epsilon^{+} \mathbb{D}_{+} \mathbb{X}^{\bar{r}}+\overline{\tilde{h}}\left(\mathbb{X}^{\bar{r}}\right) \bar{\epsilon}^{+} \overline{\mathbb{D}}_{+} \mathbb{X}^{\bar{r}}
\end{aligned}
$$

leads to invariance and closure of the algebra only on-shell and provided that the geometry is hyperkähler. The on-shell requirement follows from

$$
\left[\delta_{1}, \delta_{2}\right] \ell=-\epsilon_{[2}^{+} \bar{\epsilon}_{1]}^{+}\left|f_{\bar{\ell}}\right|^{2} \partial_{+} \cdots
$$

which has the wrong sign for supersymmetry. It is an interesting fact that on-shell closure of the algebra, together with conditions that come from invariance of the action, requires that the function $c\left(\mathbb{X}^{L}, \mathbb{X}^{R}\right)$ defined by $(2.11)$ is constant with absolute value less than one, which means that the geometry is hyperkähler. This on-shell closure is different than the one which arises in the general $(1,1)$ discussion of extended susy [8] which locates the nonclosure of the algebra to the $(+,-)$ sector where the commutator $\left[J^{(+)}, J^{(-)}\right]$multiplies the field equation. In the present case left or right susy alone require field equations.

\footnotetext{
${ }^{3}$ This gives the $\mathbb{B}$ field in a particular global gauge as $\mathbb{B}=\mathbb{B}^{(2,0)}+\mathbb{B}^{(0,2)}$ with respect to both complex structures.
} 
In [3] we argue that the ansatz for the additional supersymmetry is too restrictive and should include central charge transformations. The reason for trying to find a more general ansatz is that there is a known example of a $\mathrm{BiLP}^{4}$ with $(4,4)$ supersymmetry, mentioned in the introduction, that has a dual semichiral formulation which manifestly violates the hyperkähler condition [5]. The duality and extra supersymmetry will be discussed in detail in section 4 below. Since the isometry used in the dualisation commutes with the extra supersymmetry, the dual model is expected to have the extra symmetry as well. To investigate this, bearing in mind that the relevant algebra only closes on-shell, we now develop a novel $N=(1,1)$ form of the semichiral model and its additional symmetries.

\section{$3 \quad N=(4,4)$ in $N=(1,1)$ superspace}

We want to find out under what conditions a semichiral sigma model in $4 D$ supports additional complex structures forming an $\mathrm{SU}(2)$ algebra $^{5}$

$$
I_{(+)}^{(\mathfrak{a})} I_{(+)}^{(\mathfrak{b})}=-\delta^{\mathfrak{a} \mathfrak{b}}+\epsilon^{\mathfrak{a} \mathfrak{b} \mathfrak{c}} I_{(+)}^{(\mathfrak{c})}
$$

with $\mathfrak{a}=1,2,3$ and the identification $I_{(+)}^{(3)}:=J^{(+)}$. To this end, we discuss the situation in $(1,1)$ superspace [8]. This discussion is general, only the later applications in the example section will be limited to $4 D$. We replace spinor derivatives according to

$$
\overline{\mathbb{D}}_{ \pm} \rightarrow D_{ \pm}+i Q_{ \pm}, \quad \mathbb{D}_{ \pm} \rightarrow D_{ \pm}-i Q_{ \pm} .
$$

The general form of the $(2,2)$ sigma model reduced to $(1,1)$ has a Lagrangian that reads

$$
\mathcal{L}=D_{+} X^{A} \mathbb{E}_{A B}(X) D_{-} X^{C}+\Psi_{+}^{R} K_{R L} \Psi_{-}^{L}:=\mathcal{L}_{1}+\mathcal{L}_{2},
$$

where $\mathbb{E}_{A B}:=\mathbb{G}_{A B}+\mathbb{B}_{A B}$ and we have completed the square for the spinor auxiliary fields

$$
\psi_{+}^{R}:=Q_{+} \mathbb{X}^{R}\left|, \quad \psi_{-}^{L}:=Q_{-} \mathbb{X}^{L}\right|,
$$

(vertical bar denotes the $N=1$ component) and defined

$$
\begin{aligned}
& \Psi_{+}^{R}:=\psi_{+}^{R}-D_{+} X^{A} J_{(+) A}^{R} \\
& \Psi_{-}^{L}:=\psi_{-}^{L}-J_{(-) A}^{L} D_{-} X^{A} .
\end{aligned}
$$

Assume that we have found the additional transformations of the $(1,1)$ coordinates generated by the $\mathrm{SU}(2)$ set of complex structures $I_{(+)}^{(\mathfrak{a})}$ as in (3.1). These transformations leave the $\mathcal{L}_{1}$ part of the action invariant. We would now like to extend them to symmetries of the full action and subsequently check if the full set can come from transformations of the $(2,2)$ semichiral fields.

There are obvious symmetries we can write down, field equation symmetries (also called Zilch symmetries), but there are many possibilites.

\footnotetext{
${ }^{4}$ This acronym stands for "bihermitean local product" and refers to a $2 D$ sigma model with chiral and twisted chiral superfields only.

${ }^{5}$ Corresponding to $(+)$-supersymmetries. The general case also involves (-)-supersymmetries.
} 


\section{1 $N=(2,2)$}

To get a guide to the correct form, we use the fact that we know that one of the symmetries has the correct properties; the one generated by $I_{(+)}^{(3)}=J_{(+)}$.

The full action is invariant under the following transformations:

$$
\begin{aligned}
\delta X^{L} & =\epsilon^{+} J D_{+} X^{L} \\
\delta X^{R} & =\epsilon^{+} \psi_{+}^{R} \\
\delta \psi_{-}^{L} & =\epsilon^{+} J D_{+} \psi_{-}^{L} \\
\delta \psi_{+}^{R} & =\epsilon^{+} D_{+}^{2} X^{R}
\end{aligned}
$$

where $J$ is the canonical complex structure $\operatorname{diag}(i,-i)$. They give

$$
\begin{aligned}
\delta \mathcal{L}= & -2 \delta X^{A} \nabla_{+}^{(-)} D_{-} X^{B} \mathbb{G}_{A B}+\delta \Psi_{+}^{R} K_{R L} \Psi_{-}^{L}+\Psi_{+}^{R} \delta K_{R L} \Psi_{-}^{L}+\Psi_{+}^{R} K_{R L} \delta \Psi_{-}^{L} \\
= & -2 \epsilon^{+} J_{(+) C}^{A} D_{+} X^{C} \nabla_{+}^{(-)} D_{-} X^{B} \mathbb{G}_{A B}+\Psi_{+}^{R}\left(K_{R L} \delta \Psi_{-}^{L}+2 \epsilon^{+} \nabla_{+}^{(-)} D_{-} X^{B} \mathbb{G}_{R B}\right) \\
& +\delta \Psi_{+}^{R} K_{R L} \Psi_{-}^{L}+\Psi_{+}^{R} \delta K_{R L} \Psi_{-}^{L},
\end{aligned}
$$

where capital letters from the beginning of the alphabet takes on all values $L$ and $R$. The first of the terms in the last line is the variation of $\mathcal{L}_{1}$ under the $J_{(+)}^{(3)}$ symmetry and vanishes in the action. We evaluate the remaining terms using

$$
\begin{aligned}
\delta \Psi_{-}^{L}= & \epsilon^{+} J D_{+} \Psi_{-}^{L}+\epsilon^{+} J_{(-) R^{\prime}}^{L} D_{-} \Psi_{+}^{R^{\prime}}-\epsilon^{+} J_{(-) A R^{\prime}}^{L} \Psi_{+}^{R^{\prime}} D_{-} X^{A} \\
& +\epsilon^{+}\left[J_{(+)}, J_{(-)}\right]_{A}^{L}\left(D_{+} D_{-} X^{A}+D_{+} X^{B} \Gamma_{B C}^{(-)}{ }^{A} D_{-} X^{C}\right) \\
\delta \Psi_{+}^{R}= & \epsilon^{+} J_{(+) R^{\prime}}^{R} D_{+} \Psi_{+}^{R^{\prime}}-\epsilon^{+} J_{(+) A R^{\prime}}^{R} \Psi_{+}^{R^{\prime}} D_{+} X^{A},
\end{aligned}
$$

which follows from (3.6). (Here $J_{(-) A R}^{L}$ denotes the derivative $\left(J_{(-) A}^{L}\right), R$ etc.) Since

$$
\mathbb{G}_{A B}=\Omega_{A C}\left[J_{(+)}, J_{(-)}\right]_{B}^{C}
$$

the second line may be rewritten as

$$
\epsilon^{+} \sigma^{L C} \mathbb{G}_{C A} \nabla_{+}^{(-)} D_{-} X^{A}
$$

where we use the notation $\sigma^{L C} \Omega_{C D}=\delta_{D}^{C}$. This part of the variation will cancel the covariant derivative term multiplying $\Psi_{+}^{R}$ in (3.7). We are left with

$$
\delta \mathcal{L}=-\frac{1}{2} \epsilon^{+} D_{-}\left(\Psi_{+}^{R} C_{R R^{\prime}} \Psi_{+}^{R^{\prime}}\right)+\epsilon^{+} D_{+}\left(\Psi_{+}^{R} K_{R L} J \Psi_{-}^{L}\right),
$$

which ensures invariance of the action. Here where $C_{R R^{\prime}}:=\left[J, K_{R R^{\prime}}\right]$ is the commutator with the canonical complex structure $J$ :

$$
J:=\left(\begin{array}{cc}
i & 0 \\
0 & -i
\end{array}\right) .
$$

In deriving (3.11) heavy use is made of integrability and covariant constancy of $J_{( \pm)}$ as well as their explicit expressions [1]. 
We note that the transformations that leave the action invariant may be written

$$
\begin{aligned}
\delta X^{A}= & \epsilon^{+} J_{(+) B}^{A} D_{+} X^{B}+\delta_{R}^{A} \epsilon^{+} \Psi_{+}^{R} \\
\delta \Psi_{-}^{L}= & \epsilon^{+} J D_{+} \Psi_{-}^{L}+\epsilon^{+} J_{(-) R^{\prime}}^{L} D_{-} \Psi_{+}^{R^{\prime}}-\epsilon^{+} J_{(-) A R^{\prime}}^{L} \Psi_{+}^{R^{\prime}} D_{-} X^{A} \\
& -2 \epsilon^{+} K^{L R_{\mathbb{G}_{R A}} \nabla_{+}^{(-)} D_{-} X^{A}} \\
\delta \Psi_{+}^{R}= & \epsilon^{+} J_{(+) R^{\prime}}^{R} D_{+} \Psi_{+}^{R^{\prime}}-\epsilon^{+} J_{(+) A R^{\prime}}^{R} \Psi_{+}^{R^{\prime}} D_{+} X^{A}
\end{aligned}
$$

\section{$3.2 N=(4,4)$}

We now investigate if our additional supersymmetries (4.31) can be written in terms of transformations of semichiral fields in $(2,2)$ superspace. In our $(1,1)$ language, the relation to semichirals is given by $(3.5)$ in $\left(X^{L}, X^{R}\right)$ coordinates. ${ }^{6}$ We denote a generic complex structure by $I_{B}^{A}$ and write the $X$ transformations as

$$
\delta X^{A}=\epsilon^{+}\left[I_{(+) B}^{A} D_{+} X^{B}+M_{R}^{A} \Psi_{+}^{R}\right],
$$

where $\mathcal{L}_{1}$ is assumed to be invariant (up to total derivatives) under the first transformation on the r.h.s. Note that $M_{L}^{A}=0$. The formula (3.14) is of the form in (3.13) and the most general expression compatible with dimensions and symmetries. From (3.5) we have that

$$
\begin{aligned}
\delta \Psi_{+}^{\dot{R}}=\epsilon^{+} & {\left[\left(I_{(+) R}^{\dot{R}}-\left[M, J_{(+)}\right]_{R}^{\dot{R}}\right) D_{+} \Psi_{+}^{R}+\left(I_{+A, R}^{\dot{R}}+\mathcal{M}\left(M, J_{(+)}\right)_{R A}^{\dot{R}}\right) D_{+} X^{A} \Psi_{+}^{R}\right.} \\
& \left.-M_{R, R^{\prime}}^{\dot{R}} \Psi_{+}^{R^{\prime}} \Psi_{+}^{R}\right] \\
\delta \Psi_{-}^{\dot{L}}=\epsilon^{+} & -\left[M, J_{(-)}\right]_{R}^{\dot{L}} D_{-} \Psi_{+}^{R}+\mathcal{M}\left(M, J_{(-)}\right)_{R A}^{\dot{L}} D_{-} X^{A} \Psi_{+}^{R} \\
& +\left(I_{(+) L}^{\dot{L}}-M_{R}^{\dot{L}} J_{(+) L}^{R}\right) D_{+} \Psi_{-}^{L}+\left(I_{(+) A, L}^{\dot{L}}-M_{R}^{\dot{L}} J_{(+) A, L}^{R}\right) D_{+} X^{A} \Psi_{-}^{L} \\
& \left.-M_{R, L}^{\dot{L}} \Psi_{-}^{L} \Psi_{+}^{R}+\left(\left[I_{(+)}, J_{(-)}\right]_{A}^{\dot{L}}-M_{R}^{\dot{L}}\left[J_{(+)}, J_{(-)}\right]_{A}^{R}\right) \nabla_{+}^{(-)} D_{-} X^{A}\right]
\end{aligned}
$$

where the Magri-Morosi concomitant for two endomorphisms $I$ and $J$ reads $^{7}$

$$
\mathcal{M}(I, J)_{B D}^{A}=I_{B}^{F} J_{D, F}^{A}-J_{D}^{F} I_{B, F}^{A}-I_{F}^{A} J_{D, B}^{F}+J_{F}^{A} I_{B, D}^{F}
$$

When $M_{R}^{A}=\delta_{R}^{A}$ and $I_{+}=J_{(+)}$these transformations reduce to (3.13).

From invariance of (3.3), we find a number of relations. First, raising and lowering indices on $M$ with $K_{R L}$,

$$
\begin{aligned}
M_{L[R, \dot{R}]}-M_{[R \dot{R}], L} & =0 \\
M_{[R \dot{R}]} & =-\frac{1}{2} K_{\dot{R} \dot{L}}\left[I_{(+)}, J_{(-)}\right]_{A}^{\dot{L}} \mathbb{G}^{A L} K_{L R} \\
M_{\dot{R}}^{R} & =-\frac{1}{2} K_{\dot{R} L}\left[I_{(+)}, J_{(-)}\right]_{A}^{L} \mathbb{G}^{A R}
\end{aligned}
$$

\footnotetext{
${ }^{6}$ The reduction of $\left(\mathbb{X}^{L}, \mathbb{X}^{R}\right)$.

${ }^{7}$ Originally defined for a Poisson structure $P$ and a Nijenhuis tensor $N$ when it reads [10]

$$
C_{m}^{k j}=P^{l j} N_{m, l}^{k}+P^{k l} N_{m, l}^{k}-N_{m}^{l} P_{, l}^{k j}+N_{l}^{j} P_{, m}^{k l}-P^{l j} N_{l, m}^{k}
$$

and is only a tensor when $[P, N]=0$.
} 
Note that only the antisymmetric part of $M_{R \dot{R}}$ is determined by this. ${ }^{8}$ The $D_{-}$terms in the variation of $\mathcal{L}$ are

$$
\Psi_{+}^{\dot{R}} K_{\dot{R} L} \epsilon^{+}\left(-\left[M, J_{(-)}\right]_{R}^{L} D_{-} \Psi_{+}^{R}+\mathcal{M}\left(M, J_{(-)}\right)_{R A}^{L} D_{-} X^{A} \Psi_{+}^{R}\right)
$$

For this to yield a total $D_{-}$derivative, we shall need

$$
-K_{(\dot{R}|L|}\left[M, J_{(-)}\right]_{R)}^{L}=[J, M]_{(\dot{R} R)}+C_{(\dot{R}|R|} M_{R)}^{R}=0
$$

and

$$
K_{[\dot{R}|L|} \mathcal{M}\left(M, J_{(-)}\right)_{R] A}^{L} D_{-} X^{A}=-\frac{1}{2} D_{-}\left(K_{[\dot{R}|L|}\left[M, J_{(-)}\right]_{R]}^{L}\right) .
$$

Similarily, for the $D_{+}$terms to yield a total derivative, we need

$$
K_{L R} I_{\dot{R}}^{R}-K_{\dot{R} L} I_{L}^{L}+K_{L R}[\tilde{J}, M]_{\dot{R}}^{R}+C_{L L} K^{L R} M_{[R \dot{R}]}=0
$$

where we have dropped the plus index on $I$ and $(\tilde{J})_{\dot{R}}^{R}:=K^{R R^{\prime}} J K_{R^{\prime}} \dot{R}$. We also need

$$
\begin{aligned}
& \left(I_{A, \dot{R}}^{R}+\mathcal{M}\left(M, J_{(+)}\right)_{\dot{R} A}^{R}\right) K_{R L}+K_{\dot{R} L B} I_{A}^{B}+K_{\dot{R} \dot{L}}\left(I_{A, L}^{\dot{L}}-M_{R}^{\dot{L}} J_{(+) A, L}^{R}\right) \\
& \quad=\left(\left(I_{L}^{\dot{L}}-M_{R}^{\dot{L}} J_{(+) L}^{R}\right) K_{\dot{L} \dot{R}}\right)_{, A}
\end{aligned}
$$

where we have used (3.17) and the explicit form of $J_{(+)}$.

\section{The $S^{3} \times S^{1}$ model}

\subsection{Duality}

In this section we briefly recapitulate the dualisation of the BiLP formulation of the $\mathrm{SU}(2) \times$ $\mathrm{U}(1)$ WZW model [5], albeit in a different version.

We start from the following BiLP potential which gives a sigma model with target space geometry $S^{3} \times S^{1}$;

$$
K=-\ln \hat{\chi} \ln \hat{\bar{\chi}}+\int^{\frac{\hat{\phi} \hat{\phi}}{\tilde{\chi}}} d q \frac{\ln (1+q)}{q}
$$

where $\hat{\phi}$ is chiral, $\overline{\mathbb{D}}_{ \pm} \hat{\phi}=0$, and $\hat{\chi}$ is twisted chiral, $\overline{\mathbb{D}}_{+} \hat{\chi}=0=\mathbb{D}_{-} \hat{\chi}$. The potential satisfies the Laplacian

$$
K_{\hat{\phi} \hat{\bar{\phi}}}+K_{\hat{\chi} \hat{\bar{\chi}}}=0
$$

and hence the model has $(4,4)$ supersymmetry [8]. Changing coordinates to new chiral and twisted chiral fields, $\phi=\ln \hat{\phi}, \chi=\ln \hat{\chi}$, results in

$$
K \rightarrow K=-\chi \bar{\chi}+\int^{\phi+\bar{\phi}-\chi-\bar{\chi}} d q \ln \left(1+e^{q}\right)
$$

\footnotetext{
${ }^{8}$ The r.h.s. of the equation containing $M_{[R \dot{R}]}$ is antisymmetric due to hermiticity conditions.
} 
and makes it amenable to dualisation of the translation symmetry ${ }^{9}$

$$
\phi \rightarrow \phi+\lambda, \quad \chi \rightarrow \chi+\lambda .
$$

To apply the gauging prescription of [13] we add a term $\alpha(\chi-\bar{\chi})(\phi-\bar{\phi})$, which represents a constant $B$-term, to the Lagrangian and rewrite the potential, up to generalized Kähler gauge transformations, as

$$
\frac{1}{2}(\chi-\bar{\chi})^{2}+\alpha(\chi-\bar{\chi})(\phi-\bar{\phi})+\int^{\phi+\bar{\phi}-\chi-\bar{\chi}} d q \ln \left(1+e^{q}\right)
$$

Following [13], we find the first order action (in a WZ gauge);

$$
-\frac{1}{2} V_{\chi}^{2}-\alpha V_{\chi} V_{\phi}-V^{\prime} X^{\prime}-V_{\phi} X_{\phi}-V_{\chi} X_{\chi}+\int^{V^{\prime}} d q \ln \left(1+e^{q}\right),
$$

where $V_{\phi}, V_{\chi}$ and $V^{\prime}$ are the Large Vector Multiplet (LVM) fields [13], ${ }^{10}$ and the Lagrange multipliers are combinations of semichiral fields

$$
\begin{aligned}
X_{\phi} & =\frac{i}{2}(\ell-\bar{\ell}-r+\bar{r}) \\
X_{\chi} & =\frac{i}{2}(-\ell+\bar{\ell}-r+\bar{r}) \\
X^{\prime} & =\frac{1}{2}(\ell+\bar{\ell}-r-\bar{r}) .
\end{aligned}
$$

Eliminating the LVM and massaging the integral we find the dual semichiral action in the form

$$
-\frac{1}{2 \alpha^{2}} X_{\phi}^{2}+\frac{1}{\alpha} X_{\phi} X_{\chi}-\int^{X^{\prime}} d q \ln \left(e^{q}-1\right) .
$$

This is the potential that is expected to have additional supersymmetries due to those of the dual BiLP model.

\subsection{The geometry}

The reduction of a semichiral model to $(1,1)$ superspace may be expressed in several useful coordinate systems. E.g., the $\left(X^{L}, X^{R}\right)$ coordinates directly obtained in the reduction is related to the $(X, Y)$ system where $J_{(+)}$is canonical, $J_{(+)}=\operatorname{diag}(J, J)$, via a coordinate transformation $[1,14]$.

We now derive the metric in $(X, Y)$ coordinates for (4.8). To this end we first calculate the various ingredient matrices according to the formulae in [1].

\footnotetext{
${ }^{9}$ This is equivalent to dualising the scaling symmetry of (4.1). These isometries both commute with the extra supersymmetry $[11,12]$.

${ }^{10}$ In pure gauge they become

$$
\begin{aligned}
V_{\phi} & =i(\bar{\phi}-\phi) \\
V_{\chi} & =i(\bar{\chi}-\chi) \\
V^{\prime} & =\phi+\bar{\phi}-\chi-\bar{\chi} .
\end{aligned}
$$
}


Without loss of generality, we set $\alpha$ to -1 in (4.8) and drop the prime on $X^{\prime}$. The $Y$ coordinates are defined to be $K_{L}=: Y$. We find

$$
\begin{aligned}
-y & =K_{\ell}=\frac{i}{2} X_{\chi}+\frac{1}{2} \ln \left(e^{X}-1\right)=\frac{1}{4}(\ell-\bar{\ell})+\frac{1}{4}(r-\bar{r})+\frac{1}{2} \ln \left(e^{X}-1\right) \\
& \Rightarrow \\
X & =\ln \left(1+e^{-(y+\bar{y})}\right) \\
r+\bar{r} & =\ell+\bar{\ell}-2 \ln \left(1+e^{-(y+\bar{y})}\right), \\
r-\bar{r} & =-2(y-\bar{y})-(\ell-\bar{\ell})
\end{aligned}
$$

The relevant matrices of derivatives of $K$ are

$$
\begin{aligned}
K_{L L} & =-\frac{1}{4 N}\left(\begin{array}{cc}
E & 1 \\
1 & E
\end{array}\right), & -K_{R R} & =\frac{1}{4 N}\left(\begin{array}{cc}
2-E & M \\
4 N+1 & 2-E
\end{array}\right) \\
-K_{L R} & =-\frac{1}{4 N}\left(\begin{array}{cc}
1 & E \\
E & 1
\end{array}\right), & -K^{R L} & =\frac{1}{e^{X}}\left(\begin{array}{cc}
1 & -E \\
-E & 1
\end{array}\right) \\
-C_{L L} & =\frac{2 i}{4 N}\left(\begin{array}{cc}
0 & 1 \\
-1 & 0
\end{array}\right), & -C_{R R} & =\frac{2 i M}{4 N}\left(\begin{array}{cc}
0 & 1 \\
-1 & 0
\end{array}\right)
\end{aligned}
$$

where we have introduced the notation

$$
N:=e^{-(y+\bar{y})}=e^{X}-1, \quad E:=2 e^{X}-1, \quad M:=4 N+1,
$$

for combinations that will occur frequently in our formulae. The metric and $B$-field in $(X, Y)$ coordinates can be calculated from the formulae in [1]:

$$
\begin{aligned}
-\mathbb{E}_{L L} & =J\left(K_{L L} K^{L R} J K_{R L}-K_{L R} J K^{R L} K_{L L}-K_{L L} K^{L R} C_{R R} K^{R L} K_{L L}\right)=-2 \sigma_{1} \\
\mathbb{E}_{L Y} & =J\left(K_{L R} J K^{R L}+K_{L L} K^{L R} C_{R R} K^{R L}\right)=-\frac{1}{e^{X}}\left(\begin{array}{cc}
2-e^{X} & -2 E \\
-2 E & 2-e^{X}
\end{array}\right) \\
\mathbb{E}_{Y L} & =J\left(-K^{L R} J K_{R L}+K^{L R} C_{R R} K^{R L} K_{L L}\right)=\frac{1}{e^{X}}\left(\begin{array}{cc}
2-e^{X} & 2 E \\
2 E & 2-e^{X}
\end{array}\right) \\
-\mathbb{E}^{Y Y} & =-J K^{L R} C_{R R} K^{R L}=-\frac{2 M}{e^{X}} \sigma_{1} .
\end{aligned}
$$

Note that the tensor $\mathbb{E}$ depends on $y+\bar{y}$ only. From the formulae for $\mathbb{E}=\mathbb{G}+\mathbb{B}$, it follows that the metric is ${ }^{11}$

$$
\mathbb{G}=\frac{2}{e^{X}}\left(\begin{array}{cc}
e^{X} & E \\
E & M
\end{array}\right) \otimes \sigma_{1},
$$

with inverse

$$
\mathbb{G}^{-1}=-\frac{e^{X}}{2 N}\left(\begin{array}{cc}
-M & E \\
E & -e^{X}
\end{array}\right) \otimes \sigma_{1}=:-\frac{e^{X}}{2 N} \mathrm{~h} \otimes \sigma_{1}
$$

\footnotetext{
${ }^{11}$ Since a lot of the objects have $2 D$ complex submatrices, it is convenient to introduce the Pauli matrices $\sigma_{i}$ and write matrices as direct products.
} 
Also

$$
\mathbb{B}_{Y L}=\left(2 e^{-X}-1\right) \mathbb{1} .
$$

In these coordinates $J_{(+)}$is canonical while

$$
\begin{aligned}
J_{(-)} & =e^{-X}\left(\begin{array}{cc}
\left(2-e^{X}\right) J-2 E \sigma_{2} & -2 M \sigma_{2} \\
2 e^{X} \sigma_{2} & \left(2-e^{X}\right) J+2 E \sigma_{2}
\end{array}\right) \\
& =: e^{-X}\left(2 \mathrm{j} \otimes \sigma_{2}+\left(2-e^{X}\right) \otimes i \sigma_{3}\right),
\end{aligned}
$$

where we use that $J=i \sigma_{3}$.

\subsection{Additional SUSY in $(1,1)$}

\subsubsection{Deriving the transformations on the $(1,1)$ coordinate fields}

In the original BiLP, (4.1) the additional super symmetries read [8]

$$
\begin{aligned}
& \delta \hat{\phi}=\bar{\epsilon}^{+} \overline{\mathbb{D}}_{+} \hat{\bar{\chi}}+\bar{\epsilon}^{-} \overline{\mathbb{D}}_{-} \hat{\chi} \\
& \delta \hat{\bar{\phi}}=\epsilon^{+} \mathbb{D}_{+} \hat{\chi}+\epsilon^{-} \mathbb{D}_{-} \hat{\bar{\chi}} \\
& \delta \hat{\chi}=-\bar{\epsilon}^{+} \overline{\mathbb{D}}_{+} \hat{\bar{\phi}}-\epsilon^{-} \mathbb{D}_{-} \hat{\phi} \\
& \delta \hat{\bar{\chi}}=-\epsilon^{+} \mathbb{D}_{+} \hat{\phi}-\bar{\epsilon}^{-} \overline{\mathbb{D}}_{-} \hat{\bar{\phi}},
\end{aligned}
$$

and in the transformed version (4.3) they become

$$
\begin{aligned}
& \delta \phi=e^{\bar{\chi}-\phi} \bar{\epsilon}^{+} \overline{\mathbb{D}}_{+} \bar{\chi}+e^{\chi-\phi} \bar{\epsilon}^{-} \overline{\mathbb{D}}_{-} \chi \\
& \delta \bar{\phi}=e^{\chi-\bar{\phi}} \epsilon^{+} \mathbb{D}_{+} \chi+e^{\bar{\chi}-\bar{\phi}} \epsilon^{-} \mathbb{D}_{-} \bar{\chi} \\
& \delta \chi=-e^{\bar{\phi}-\chi} \bar{\epsilon}^{+} \overline{\mathbb{D}}_{+} \bar{\phi}-e^{\phi-\chi} \epsilon^{-} \mathbb{D}_{-} \phi \\
& \delta \bar{\chi}=-e^{\phi-\bar{\chi}} \epsilon^{+} \mathbb{D}_{+} \phi-e^{\bar{\phi}-\bar{\chi}_{\bar{\epsilon}}-\overline{\mathbb{D}}_{-} \bar{\phi}} .
\end{aligned}
$$

These relations survive in the $(1,1)$ reduction with $\mathbb{D}_{ \pm} \rightarrow D_{ \pm}$. From $(4.17)$ we then read off the additional complex structures according to

$$
\delta \varphi=\frac{1}{2}\left[\left(J_{( \pm)}^{(1)}+i J_{( \pm)}^{(2)}\right) \epsilon^{ \pm} D_{ \pm} \varphi+\left(J_{( \pm)}^{(1)}-i J_{( \pm)}^{(2)}\right) \bar{\epsilon}^{ \pm} D_{ \pm} \varphi\right]
$$

For the $J_{(+)}^{(\mathfrak{a})}$ we find

$$
\begin{aligned}
J_{(+)}^{(1)} & =\left(\begin{array}{cccc}
0 & 0 & 0 & e^{\bar{\chi}-\phi} \\
0 & 0 & e^{\chi-\bar{\phi}} & 0 \\
0 & -e^{\bar{\phi}-\chi} & 0 & 0 \\
-e^{\phi-\bar{\chi}} & 0 & 0 & 0
\end{array}\right) \\
J_{(+)}^{(2)} & =\left(\begin{array}{cccc}
0 & 0 & 0 & i e^{\bar{\chi}-\phi} \\
0 & 0 & -i e^{\chi-\bar{\phi}} & 0 \\
0 & -i e^{\bar{\phi}-\chi} & 0 & 0 \\
i e^{\phi-\bar{\chi}} & 0 & 0 & 0
\end{array}\right),
\end{aligned}
$$

with $J_{(+)}^{(3)}=J$, the canonical complex structure. 
We would like to see what these complex structures look like in $(1,1)$ coordinates related to the semichiral description. While T-dual formulations are not in general related by coordinate transformations, they are in this case due to the special choice of the isometry direction; we have dualised along the common $\mathrm{U}(1)$. We now need to find the coordinate transformation. To this end, we note that the relations (A.5), derived in the appendix,

$$
\begin{aligned}
V_{\phi} & =X_{\chi}+X_{\phi} \\
V_{\chi} & =X_{\phi} \\
V & =\ln \left(e^{X}-1\right) .
\end{aligned}
$$

do not completely determine the transformations. Identifying the 1.h.s. with BiLP fields (WZ-gauge) and writing out the r.h.s. we have

$$
\begin{aligned}
i(\bar{\phi}-\phi) & =-i(r-\bar{r}) \\
i(\bar{\chi}-\chi) & =\frac{i}{2}(\ell-\bar{\ell}-r+\bar{r}) \\
\phi+\bar{\phi}-\chi-\bar{\chi} & =\ln \left(e^{\frac{1}{2}(\ell+\bar{\ell}-r-\bar{r})}-1\right) .
\end{aligned}
$$

It turns out to be most convenient to identify the coordinate transformation to $(X, Y)$ coordinates where the complex structure derived from the semi side, $J_{(+)}$, is canonical. ${ }^{12}$ A coordinate transformation to $\left(X^{L}, X^{R}\right)$ coordinates will then give a non-canonical $J_{(+)}$. Comparing this to (4.9), we identify

$$
\begin{aligned}
y & =\chi-\phi \\
\ell-\bar{\ell} & =2(\bar{\chi}-\chi)-(\bar{\phi}-\phi),
\end{aligned}
$$

but $\ell+\bar{\ell}$ is left undetermined. In both coordinate systems we have $J_{(+)}^{(3)}=J$. Requiring that the coordinate transformation takes the canonical complex structure into itself ${ }^{13}$ determines $\ell+\bar{\ell}=\phi+\bar{\phi}-2(\bar{\chi}-\chi)$, which results in

$$
\ell=\phi-2 \chi
$$

This gives the transformation Jacobian

$$
\Lambda=\left(\begin{array}{ll}
\frac{\partial L}{\partial \phi} & \frac{\partial L}{\partial \chi} \\
\frac{\partial Y}{\partial \phi} & \frac{\partial Y}{\partial \chi}
\end{array}\right)=\left(\begin{array}{cc}
\mathbb{1} & -2 \mathbb{1} \\
-\mathbb{1} & \mathbb{1}
\end{array}\right),
$$

with inverse

$$
\Lambda^{-1}=-\left(\begin{array}{cc}
\mathbb{1} & 2 \mathbb{1} \\
\mathbb{1} & \mathbb{1}
\end{array}\right),
$$

\footnotetext{
${ }^{12}$ The map of $J_{( \pm)}$under duality is discussed in [15] where the dual model appears in some preferred coordinates. We have not investigated the relation to the present coordinates.

${ }^{13}$ This condition requires some comments. A coordinate transformation preserves the commutator between matrices. Hence if we also identify the semi $J_{(-)}$as the coordinate transformation of the BiLP $J_{(-)}$we would conclude that $\left[J_{(+)}, J_{(-)}\right]=0$ on the semi side, which is incorrect. The strategy here is to assume that there is an $\mathrm{SU}(2)$ of complex structures on the semi side containing the semi $J_{(+)}$and that the $\operatorname{BiLP} J_{(+)}$is mapped into the latter. The map of the BiLP $J_{(-)}$is not the semi $J_{(-)}$but another complex structure that commutes with the semi $J_{(+)}$and which is not considered in what follows.
} 
These transformations correctly relates the BiLP metric derived from (4.3) to the semi metric (4.12). We now write the extra complex structures $J_{(+)}^{(\mathfrak{a})}$ as

$$
J_{(+)}^{(\mathfrak{a}))}=\left(\begin{array}{cc}
0 & \mathbb{A}^{(\mathfrak{a})} \\
-\left(\mathbb{A}^{(\mathfrak{a})}\right)^{-1} & 0
\end{array}\right)
$$

for $\mathfrak{a}=1,2$, with

$$
\begin{aligned}
\mathbb{A}^{(1)} & =\left(\begin{array}{cc}
0 & e^{\bar{\chi}-\phi} \\
e^{\chi-\bar{\phi}} & 0
\end{array}\right)=\left(\begin{array}{cc}
0 & e^{\varphi+y} \\
e^{-\varphi+\bar{y}} & 0
\end{array}\right) \\
\mathbb{A}^{(2)} & =\left(\begin{array}{cc}
0 & i e^{\bar{\chi}-\phi} \\
-i e^{\chi-\bar{\phi}} & 0
\end{array}\right)=\left(\begin{array}{cc}
0 & i e^{\varphi+y} \\
-i e^{-\varphi+\bar{y}} & 0
\end{array}\right),
\end{aligned}
$$

where

$$
\varphi:=(\ell-\bar{\ell})+(y-\bar{y}) .
$$

The expressions for $J_{(+)}^{(\mathfrak{a})}$ in $(X, Y)$ coordinates then become

$$
\begin{aligned}
J_{(+)}^{(\mathfrak{a})} & =\left(\begin{array}{cc}
-E & -M \\
e^{X} & E
\end{array}\right) \otimes \mathbb{A}^{(\mathfrak{a})}=: j \otimes \mathbb{A}^{(\mathfrak{a})}, \\
j^{2} & =-N, \quad\left(\mathbb{A}^{(\mathfrak{a})}\right)^{2}=N^{-1},
\end{aligned}
$$

where we again use the notation in (4.11). The complex structures $J_{(+)}^{(\mathfrak{a})}$ preserve the metric (4.12), as confirmed by an explicit calculation.

\subsection{2 $\left(X^{L}, X^{R}\right)$ coordinates}

Using (4.10) in the Jacobian

$$
\begin{aligned}
\Lambda & =\left(\begin{array}{cc}
\mathbb{1} & 0 \\
K_{L L} & K_{L R}
\end{array}\right)=\frac{1}{4 N}\left(\begin{array}{cc}
4 N & 0 \\
-\left(E \mathbb{1}+\sigma_{1}\right) & \mathbb{1}+E \sigma_{1}
\end{array}\right), \\
\Lambda^{-1} & =\left(\begin{array}{cc}
\mathbb{1} & 0 \\
-K^{R L} K_{L L} & K^{R L}
\end{array}\right)=\left(\begin{array}{cc}
\mathbb{1} & 0 \\
\sigma_{1}-e^{-X}\left(\mathbb{1}-E \sigma_{1}\right)
\end{array}\right),
\end{aligned}
$$

we find the expressions for $J_{(+)}^{(\mathfrak{a})}$ in left right coordinates $\left(X^{L}, X^{R}\right)$ :

$$
\begin{aligned}
J_{(+)}^{(\mathfrak{a})}=\frac{1}{4 N}\{ & \left(\begin{array}{cc}
E & -M \\
e^{-X} & -e^{-X} E
\end{array}\right) \otimes \mathbb{A}^{(\mathfrak{a})}+\left(\begin{array}{cc}
M & -M E \\
e^{-X} E & -e^{-X} E^{2}
\end{array}\right) \otimes \mathbb{A}^{(\mathfrak{a})} \sigma_{1} \\
& \left.+\frac{N}{e^{X}}\left[\left(\begin{array}{cc}
0 & 0 \\
1 & -E
\end{array}\right) \otimes \overline{\mathbb{A}}^{(\mathfrak{a})}+\left(\begin{array}{cc}
0 & 0 \\
E & -1
\end{array}\right) \otimes \overline{\mathbb{A}}^{(\mathfrak{a})} \sigma_{1}\right]\right\},
\end{aligned}
$$

where $Y\left(X^{L}, X^{R}\right)$ is given by the relations in (4.9). 


\section{$5 \quad$ Examples}

\subsection{Hyperkähler}

We want to show that there are hyperkähler solutions to our problem in $4 D$. To this end we note that, when $c$ is constant we have available the following hyperkähler structure [16];

$$
\mathcal{I}:=J_{(+)}, \quad \mathcal{J}:=\frac{1}{\sqrt{1-c^{2}}}\left(J_{(-)}+c J_{(+)}\right), \quad \mathcal{K}:=\frac{1}{2 \sqrt{1-c^{2}}}\left[J_{(+)}, J_{(-)}\right] .
$$

The relations (3.17) determine $M$ in the three cases according to

$$
\begin{aligned}
\mathcal{I}: & M_{\dot{R}}^{R}=\delta_{\dot{R}}^{R}, \quad M_{[\dot{R} R]}=0 \\
\mathcal{J}: & M_{\dot{R}}^{R}=\frac{c \delta_{\dot{R}}^{R}}{\sqrt{1-c^{2}}}, \\
\mathcal{K}: M_{\dot{R}}^{R} K_{L R} & =-\frac{1}{\sqrt{1-c^{2}}} K_{\dot{R} L} J_{(-) L}^{L}=-\frac{1}{\sqrt{1-c^{2}}} J K_{\dot{R} L} \\
M_{[\dot{R} R]} & =-\frac{1}{\sqrt{1-c^{2}}} K_{\dot{R} L} J_{(-) R}^{L}=-\frac{1}{\sqrt{1-c^{2}}} C_{\dot{R} R}
\end{aligned}
$$

Each case satisfies the first relation in (3.17) (provided that $c$ is constant).

The conditions (3.18) is satisfied by the hyperkähler structure (5.2). The relation (3.19) is satisfied for $\mathcal{I}$ and $\mathcal{J}$ by direct insertion. For $\mathcal{K}$ we determine the full

$$
M_{\dot{R}}^{L}=-\frac{1}{\sqrt{1-c^{2}}} K^{L R} J K_{R \dot{R}}
$$

and find that

$$
K_{[\dot{R}|L|}\left[M, J_{(-)}\right]_{R]}^{L}=0,
$$

and the issue becomes the vanishing of $\mathcal{M}$. This is again confirmed by direct insertion of the $\mathcal{K}$ expressions from (5.2).

As a final check we also find that the relations (3.20) and (3.21) are indeed satisfied for $\mathcal{I}, \mathcal{J}$ and $\mathcal{K}$.

\section{$\mathbf{5 . 2} \mathrm{SU}(2) \otimes \mathrm{U}(1)$}

Using (4.31) and (4.15) we find that in $(X, Y)$ coordinates

$$
\left[J_{+}^{(\mathfrak{a})}, J_{-}\right]=-2 e^{-X}\left(\left(2-e^{X}\right) \mathbf{j} \otimes\left(b^{(\mathfrak{a})} \sigma_{1}+a^{(\mathfrak{a})} \sigma_{2}\right)+2 N \mathbb{1} \otimes a^{(\mathfrak{a})} i \sigma_{3}\right),
$$

where $\mathrm{j}$ is defined in (4.15). Using (4.28) we have defined

$$
\begin{aligned}
& \mathbb{A}^{(1)}=: a^{(1)} \sigma_{1}-b^{(1)} \sigma_{2}=: \frac{1}{\sqrt{N}}\left(\cos \psi \sigma_{1}-\sin \psi \sigma_{2}\right) \\
& \mathbb{A}^{(2)}=: a^{(2)} \sigma_{1}-b^{(2)} \sigma_{2}=: \frac{1}{\sqrt{N}}\left(-\sin \psi \sigma_{1}-\cos \psi \sigma_{2}\right),
\end{aligned}
$$

and

$$
i \psi:=(\ell-\bar{\ell})+\frac{3}{2}(y-\bar{y})
$$


As is clear from $(3.17)$, we shall need $\left[J_{+}^{(\mathfrak{a})}, J_{-}\right] \mathbb{G}^{-1}$. We find

$$
\left[J_{+}^{(\mathfrak{a})}, J_{-}\right] \mathbb{G}^{-1}=2 a^{(A)} \mathrm{h} \otimes \sigma_{2}+\left(2-e^{X}\right)\left(\begin{array}{cc}
0 & 1 \\
-1 & 0
\end{array}\right) \otimes\left(b^{(\mathfrak{a})} \mathbb{1}-a^{(\mathfrak{a})} i \sigma_{3}\right),
$$

where $\mathrm{h}$ is defined in (4.13). In $\left(X^{L}, X^{R}\right)$ coordinates this becomes

$$
\left(2 e^{-X}-1\right)\left(\begin{array}{cc}
0 & 1 \\
-1 & 0
\end{array}\right) \otimes b^{(\mathfrak{a})}\left(\mathbb{1}-E \sigma_{1}\right)+\left(\begin{array}{cc}
2 M & E \\
E & 0
\end{array}\right) \otimes a^{(\mathfrak{a})} \sigma_{2}-\left(\begin{array}{cc}
0 & 1 \\
-1 & 0
\end{array}\right) \otimes a^{(\mathfrak{a})} i \sigma_{3} .
$$

We read off the matrices relevant to (3.17)

$$
\begin{aligned}
M_{[R \dot{R}]} & =\frac{1}{2} K_{\dot{R} \dot{L}}\left(\left[J_{+}^{(\mathfrak{a})}, J_{-}\right] \mathbb{G}^{-1}\right)^{\dot{L} L} K_{L R}=-\frac{M e^{X} a^{(A)}}{4 N} \sigma_{2} \\
M_{L R} & =\frac{1}{2} K_{R \dot{L}}\left(\left[J_{+}^{(\mathfrak{a})}, J_{-}\right] \mathbb{G}^{-1}\right)^{\dot{L} R} K_{R L} \\
& =-\frac{1}{2} \frac{e^{X}}{4 N}\left[\left(2 e^{-X}-1\right) b^{(\mathfrak{a})}\left(1+E \sigma_{1}\right)+a^{(\mathfrak{a})}\left(E \sigma_{2}-i \sigma_{3}\right)\right] .
\end{aligned}
$$

We find that the quantities in (5.10) indeed satisfy the first relation in (3.17). Proceeding to (3.18) and (3.19), we find that (3.18) is also satisfied using (5.10), and that the $b^{(\mathfrak{a})}$ terms in (3.19) cancel. However, the remaining terms in (3.19) must satisfy

$$
\begin{aligned}
\left(M_{[r}^{F} K_{\bar{r}] F}\right)_{R} & =0 \\
M_{r[, r}^{r} K_{\bar{r}] L} & =0,
\end{aligned}
$$

where knowledge of the form of $J_{(-)}$along with partial information from (5.10) has been used. While the first of these equations determines the remaining parts of $M_{R}^{L}$, the second equations must be identically satisfied by $M_{R}^{R}$ in (5.10). This is not the case.

\section{Discussion}

We have extended the $(1,1)$ formulation of semichiral sigma models to allow for a treatment of extra super symmetries with on-shell closure. To exemplify the general method we have shown that a set of hyperkähler geometries arise as solutions of the conditions for extra supersymmetry. We have further constructed the extra super symmetries in a semichiral models dual to a BiLP model with "manifest" $(4,4)$ susy on the BiLP side. This model fails the criteria for the additional supersymmetry to be manifest as transformations of $(2,2)$ semichirals. Another way of saying this is that the $(4,4)$ supersymmetry is incompatible with the introduction of the $(2,2)$ auxiliary spinor fields. The key ingredient in the analysis is to show that invariance of the action fails (on-shell closure of the algebra is ensured by construction). Note that the analysis shows that not even an extra supersymmetry of one handedness only is possible.

Our analysis is carried out at the $(1,1)$ level, where conditions for additional supersymmetries are well established since thirty years [8]. 
An analysis at the $(2,2)$ level already indicated that the remedy suggested in [3] will not work; a formulation including central charge transformations will typically display the original obstructions when we go on-shell.

A further indication of problems with an extra supersymmetry comes from dualisation procedure itself. One would expect the parent action, where the chirality constraints on the chiral and twisted chiral superfields have been relaxed, to have the extra supersymmetry. This would mean the the LVM gauge multiplet could carry extra supersymmetry. This was concluded to be impossible under fairly general assumptions in [2].

In view of this result, it is reasonable to conjecture that manifest extra supersymmetries involving semichiral fields together with a $4 D$ target space is only possible in models including auxiliary fields such as in the $(4,4)$ superspace setting of [17].

\section{Acknowledgments}

Discussions with M. Roček at various stages of this work are gratefully acknowledged. Supported in part by VR grant 621-2013-4245.

\section{A Duality in $(1,1)$}

In this section we reduce the action (4.6) (with $\alpha=-1$ ) to $(1,1)$ and eliminate the LVM there instead. This makes clear the issue of coordinate transformations at the $(1,1)$ level. We replace covariant derivatives according to

$$
\bar{D}_{ \pm} \rightarrow D_{ \pm}+i Q_{ \pm}, \quad D_{ \pm} \rightarrow D_{ \pm}-i Q_{ \pm}
$$

To facilitate the calculation we introduce the following notation:

$$
\begin{aligned}
Y_{ \pm}^{A} & :=Q_{ \pm} X_{A}, & & \\
Z^{A} & :=Q_{+} Q_{-} X_{A}, & & A=\phi, \chi, X \\
s^{\ell} & :=\ell+\bar{\ell}, & & d^{\ell}:=\ell-\bar{\ell} \\
s^{r} & :=r+\bar{r}, & & d^{r}:=r-\bar{r} \\
\Sigma & :=\psi+\bar{\psi} & & \\
\Lambda & :=\psi-\bar{\psi} & &
\end{aligned}
$$

and define the $(1,1)$ components of the LVM (in WZ gauge) as

$$
\begin{aligned}
V_{\chi} \mid & =: V_{\chi}, & & Q_{ \pm} V_{\chi} \mid=:(A+B)_{ \pm}, & & Q_{+} Q_{ \pm} V_{\chi} \mid=: F \\
V_{\phi} \mid & =: V_{\phi}, & Q_{ \pm} V_{\phi} \mid & =: B_{ \pm}, & & Q_{+} Q_{ \pm} V_{\phi} \mid=: G \\
V_{X} \mid & =: V_{X}, & Q_{ \pm} V_{X} \mid & =: C_{ \pm}, & & Q_{+} Q_{ \pm} V_{X} \mid=: H .
\end{aligned}
$$


The Lagrangian becomes

$$
\begin{aligned}
F & \left.V_{\phi}-V_{\chi}-X_{\chi}\right)+G\left(V_{\chi}-X_{\phi}\right)+H\left(\ln \left(1+e^{V}\right)-X\right) \\
& -V_{\phi} Z^{\phi}-V_{\chi} Z^{\chi}-V^{\prime} Z^{X}-\left(A_{+}+Y_{+}^{\chi}\right)\left(A_{-}+Y_{-}^{\chi}\right)+Y_{+}^{\chi} Y_{-}^{\chi} \\
& +\left(B_{+}-Y_{+}^{\phi}-Y_{+}^{\chi}\right)\left(B_{-}+Y_{-}^{\phi}+Y_{-}^{\chi}\right)-\left(Y_{+}^{\phi}+Y_{+}^{\chi}\right)\left(Y_{-}^{\phi}+Y_{-}^{\chi}\right) \\
& +\left(C_{+}-Y_{+}^{X}\left(\frac{1+e^{V}}{e^{V}}\right)\right)\left(\frac{e^{V}}{1+e^{V}}\right)\left(C_{-}-\left(\frac{1+e^{V}}{e^{V}}\right) Y_{-}^{X}\right)-Y_{+}^{X} Y_{-}^{X}\left(\frac{1+e^{V}}{e^{V}}\right) .
\end{aligned}
$$

Integrating out $F, G, H$ gives the coordinate transformation

$$
\begin{aligned}
V_{\phi} & =X_{\chi}+X_{\phi} \\
V_{\chi} & =X_{\phi} \\
V & =\ln \left(e^{X}-1\right) .
\end{aligned}
$$

Integrating $V_{\phi}, V_{\chi}, V$ determines $F, G, H$ in terms of the components of the semis:

$$
\begin{aligned}
& F=Z^{\phi} \\
& G=Z^{\phi}+Z^{\chi} \\
& H=\left(\frac{1+e^{V}}{e^{V}}\right)\left[\left(1-\frac{1+e^{V}}{e^{V}}\right) Y_{+}^{X} Y_{-}^{X}+Z^{X}\right] .
\end{aligned}
$$

(No contribution from $C_{ \pm}$terms etc. 1.5 formalism.) Finally, integrating $A, B, C$ again determines these fields in terms of the semi components. This leaves us with a purely semi Lagrangian;

$$
Y_{+}^{\chi} Y_{-}^{\chi}-\left(Y_{+}^{\phi}+Y_{+}^{\chi}\right)\left(Y_{-}^{\phi}+Y_{-}^{\chi}\right)-Y_{+}^{X} Y_{-}^{X}\left(\frac{e^{X}}{e^{X}-1}\right)-\left(X_{\chi}+X_{\phi}\right) Z^{\phi}-X_{\phi} Z^{\chi}-\ln \left(e^{X}-1\right) Z^{X}
$$

As a check that this agrees with the reduced semi action, we integrate out the auxiliary spinors $\Psi_{ \pm}$and reconstruct the complex structures $J^{( \pm)}$. We shall need

$$
\begin{aligned}
Y_{+}^{\phi} & =\frac{i}{2}\left[i D_{+} s^{\ell}-\Lambda_{+}^{r}\right] \\
Y_{-}^{\phi} & =\frac{i}{2}\left[\Lambda_{-}^{\ell}-i D_{-} s^{r}\right] \\
Y_{+}^{\chi} & =-\frac{i}{2}\left[i D_{+} s^{\ell}+\Lambda_{+}^{r}\right] \\
Y_{-}^{\chi} & =-\frac{i}{2}\left[\Lambda_{-}^{\ell}+i D_{-} s^{r}\right] \\
Y_{+}^{X} & =\frac{1}{2}\left[i D_{+} d^{\ell}-\Sigma_{+}^{r}\right] \\
Y_{-}^{X} & =\frac{1}{2}\left[\Sigma_{-}^{\ell}-i D_{-} d^{r}\right] \\
Z^{\phi} & =-\frac{1}{2}\left[D_{+} \Sigma_{-}^{\ell}+D_{-} \Sigma_{+}^{r}\right] \\
Z^{\chi} & =\frac{1}{2}\left[D_{+} \Sigma_{-}^{\ell}-D_{-} \Sigma_{+}^{r}\right] \\
Z^{X} & =\frac{i}{2}\left[D_{+} \Lambda_{-}^{\ell}+D_{-} \Lambda_{+}^{r}\right]
\end{aligned}
$$


From the variations we find:

$$
\begin{array}{ll}
\delta \Lambda_{+}^{r}: & \Lambda_{-}^{\ell}=3 i D_{-} s^{r}-2 i\left(\frac{e^{X}}{e^{X}-1}\right) D_{-} X \\
\delta \Sigma_{+}^{r}: & \Sigma_{-}^{\ell}=i D_{-} d^{r}-2\left(\frac{e^{X}-1}{e^{X}}\right) D_{-}\left(X_{\chi}+2 X_{\phi}\right) \\
\delta \Lambda_{-}^{\ell}: & \Lambda_{+}^{r}=-i D_{+} s^{\ell}+2 i\left(\frac{e^{X}}{e^{X}-1}\right) D_{+} X \\
\delta \Sigma_{-}^{\ell}: & \Sigma_{+}^{r}=i D_{+} d^{\ell}+2\left(\frac{e^{X}-1}{e^{X}}\right) D_{+} X_{\chi} .
\end{array}
$$

This implies that (in the notation of (4.11))

$$
\begin{aligned}
& \Psi_{-}^{\ell}=i \frac{1}{4 e^{X} N}\left[\left(1+E^{2}\right) D_{-} \ell+2 E D_{-} \bar{\ell}-2(4 N+1) E D_{-} r-2(4 N+1) D_{-} \bar{r}\right] \\
& \Psi_{+}^{r}=i \frac{1}{4 e^{X} N}\left[2 E D_{+} \ell+2 D_{+} \bar{\ell}-\left(1+E^{2}\right) D_{+} r-2 E D_{+} \bar{r}\right]
\end{aligned}
$$

These are the correct expressions for these auxiliary fermions, as may be checked using the matrices (4.10) in the formulae for $J^{( \pm)}$in [1].

\section{B An alternative dual form}

We have been studying the action (4.8). It can be cast into a different form which connects to the results in [18]. We perform a Legendre transformation of the right semichiral superfields (for $\alpha=1$ )

$$
\tilde{K}=K(L, x, \bar{x})-x r-\bar{x} \bar{r}
$$

which together with the change $L \rightarrow-L$ (and some manipulations of the integral) brings the potential to the form found in [18]:

$$
-(\ell-\bar{r})(\bar{\ell}-r)+\int^{r+\bar{r}} d q \ln \left(1+e^{q}\right) .
$$

We already know the metric in these $\left(X^{L}, X^{R}\right)$ coordinates from [18]

$$
\mathbb{G}=\left(\begin{array}{cc}
\sigma_{1} & -Z \\
-Z & Z \sigma_{1}
\end{array}\right),
$$

where

$$
Z:=\frac{1}{1+e^{r+\bar{r}}}=\frac{1}{1+e^{\ell+\bar{\ell}-y-\bar{y}}},
$$

and transformation to new $(X, Y)$ coordinates reads

$$
\begin{aligned}
& y=K_{\ell}=\bar{\ell}-r \\
& r=\bar{\ell}-y .
\end{aligned}
$$


The corresponding Jacobian is [1]

$$
J=\left(\begin{array}{cc}
\mathbb{1} & 0 \\
-K^{R L} K_{L L} & K^{R L}
\end{array}\right) .
$$

We have

$$
\begin{array}{ll}
K_{L L}=-\sigma_{1}, & K_{R R}=-Z \sigma_{1} \\
K_{L R}=\mathbb{1}, & K^{R L}=\mathbb{1}
\end{array}
$$

which implies

$$
\mathbb{G} \rightarrow\left(\begin{array}{cc}
-\sigma_{1} & 0 \\
0 & Z \sigma_{1}
\end{array}\right)
$$

Open Access. This article is distributed under the terms of the Creative Commons Attribution License (CC-BY 4.0), which permits any use, distribution and reproduction in any medium, provided the original author(s) and source are credited.

\section{References}

[1] U. Lindström, M. Roček, R. von Unge and M. Zabzine, Generalized Kähler manifolds and off-shell supersymmetry, Commun. Math. Phys. 269 (2007) 833 [hep-th/0512164] [INSPIRE].

[2] M. Göteman, U. Lindström, M. Roček and I. Ryb, Off-shell $N=(4,4)$ supersymmetry for new $(2,2)$ vector multiplets, JHEP 03 (2011) 088 [arXiv: 1008.3186] [INSPIRE].

[3] M. Göteman, U. Lindström and M. Roček, Semichiral $\sigma$-models with 4 D hyperKähler geometry, JHEP 01 (2013) 073 [arXiv:1207.4753] [INSPIRE].

[4] M. Roček, K. Schoutens and A. Sevrin, Off-shell WZW models in extended superspace, Phys. Lett. B 265 (1991) 303 [INSPIRE].

[5] U. Lindström, I. Ryb, M. Roček, R. von Unge and M. Zabzine, T-duality for the $S^{1}$ piece in the $S^{3} \times S^{1}$ model, unpublished (2009).

[6] I. Bakas and K. Sfetsos, T duality and world sheet supersymmetry, Phys. Lett. B 349 (1995) 448 [hep-th/9502065] [INSPIRE].

[7] T. Buscher, U. Lindström and M. Roček, New supersymmetric $\sigma$ models with Wess-Zumino terms, Phys. Lett. B 202 (1988) 94 [INSPIRE].

[8] S.J. Gates Jr., C.M. Hull and M. Roček, Twisted multiplets and new supersymmetric nonlinear $\sigma$-models, Nucl. Phys. B 248 (1984) 157 [INSPIRE].

[9] M. Gualtieri, Generalized complex geometry, Ph.D. Thesis, Oxford University (2003) [math.DG/0401221] [INSPIRE].

[10] F. Magri and C. Morosi, A geometrical characterization of integrable Hamiltonian systems through the theory of Poisson-Nijenhuis manifolds, Quaderni del Dipartimento di Matematica, Università di Milano (1984).

[11] P.M. Crichigno, The semi-chiral quotient, hyperKähler manifolds and T-duality, JHEP 10 (2012) 046 [arXiv:1112.1952] [INSPIRE]. 
[12] M. Göteman, $N=(4,4)$ supersymmetry and T-duality, Symmetry 4 (2012) 603 [arXiv: 1208.2166] [INSPIRE].

[13] U. Lindström, M. Roček, I. Ryb, R. von Unge and M. Zabzine, T-duality and generalized Kähler geometry, JHEP 02 (2008) 056 [arXiv:0707.1696] [INSPIRE].

[14] J. Bogaerts, A. Sevrin, S. van der Loo and S. Van Gils, Properties of semichiral superfields, Nucl. Phys. B 562 (1999) 277 [hep-th/9905141] [INSPIRE].

[15] I.T. Ivanov, B.-b. Kim and M. Roček, Complex structures, duality and WZW models in extended superspace, Phys. Lett. B 343 (1995) 133 [hep-th/9406063] [INSPIRE].

[16] M. Göteman and U. Lindström, Pseudo-hyperKähler geometry and generalized Kähler geometry, Lett. Math. Phys. 95 (2011) 211 [arXiv:0903.2376] [INSPIRE].

[17] U. Lindström, I.T. Ivanov and M. Roček, New $N=4$ superfields and $\sigma$-models, Phys. Lett. B 328 (1994) 49 [hep-th/9401091] [INSPIRE].

[18] A. Sevrin, W. Staessens and D. Terryn, The generalized Kähler geometry of $N=(2,2)$ WZW-models, JHEP 12 (2011) 079 [arXiv:1111.0551] [INSPIRE]. 